\title{
Construction and Selection of Bayesian Chain Sampling Plan (BChSP-1) Using Quality Regions
}

\author{
K.K. Suresh \\ Professor and Head \\ Department of statistics, Bharathiar University \\ Coimbatore-641046, Tamilnadu, India \\ E-mail: sureshkk1@rediffmail.com \\ V. Sangeetha \\ Ph.D Research Scholar \\ Department of statistics, Bharathiar University \\ Coimbatore-641046, Tamilnadu, India \\ E-mail: sangee.stat07@gmail.com
}

Received: November 18, 2010

Accepted: December 22, 2010

doi:10.5539/mas.v5n2p226

\begin{abstract}
Bayesian Acceptance Sampling Approach is associated with utilization of prior process history for the selection of Distributions (viz., Gamma Poisson, Beta Binomial) to describe the random fluctuations involved in Acceptance Sampling. The Acceptance sampling procedures are the practical tools for quality assurance applications involving product control. Acceptance sampling systems are advocated when small sample size are necessary or desirable towards costlier testing for product quality. The sampling plan which provides the vendor and buyer decision rules for product acceptance to meet the preset product quality requirement.

The main thrust of this paper is to account for the possibility of dependence among the items of a sample. The development of new method for designing sampling plans based on range of quality instead of point- wise description of quality by invoking a novel approach called Quality Regions. This method seems to be versatile and can be adopted to the elementary production process where the stipulated quality level is advisable to fix at a later stage and provides a new procedure meant for designing Bayesian Chain Sampling Plan involving Quality Regions. Maximum Allowable Percent Defective (MAPD) is also considered for the selection of parameters for Bayesian Chain Sampling Plan. New quality descriptors called operating ratios are introduced to design the sampling plan.
\end{abstract}

Keywords: Chain Sampling Plan, Bayesian Sampling Plan, Quality Decision Region, Probabilistic Quality Region, Limiting Quality Region, Indifference Quality Region, Maximum Allowable Percent Defective

\section{Introduction}

Classical statistics is directed towards the use of sample information. In addition to the sample information two other types of information are typically relevant. The first is knowledge of the possible consequences of the decision and the second source of non-sample information is prior information. Thomas Bayes was first to use the prior information in inductive inference and the approach to statistics, which formally seeks to utilize prior information is called Bayesian analysis. Suppose a product in a series is supplying a product, due to random fluctuations can be separated in to within lot (sampling) variations of individual units and between lot (sampling and process) variations.

Bayesian Acceptance Sampling approach is associated with the utilization of prior process history for the selection of distributions (viz., Gamma Poisson, Beta Binomial)to describe the random fluctuations involved in Acceptance Sampling. Bayesian sampling plans requires the user to specify explicitly the distribution of defectives from lot to lot. The prior distribution is the expected distribution of a lot quality on which the sampling plan is going to operate. The distribution is called prior because it is formulated prior to the taking of samples. The combination of prior knowledge, represented with the prior distribution, and the empirical knowledge based on the sample leads to the decision on the lot. 
To improve the quality for any product and services, it is customary to modernize the quality practices and simultaneously reduce the cost for inspection and quality improvement. An efficient quality improvement programme can be instrumental with increasing productivity at reduced cost. As a result of increasing customer quality requirements and development for new product technology, many existing quality assurance practices and techniques need to be modified. The need for such statistical and analytical techniques in quality assurance is rapidly increasing owing to stiff competition in industry towards product quality improvement.

This paper introduces a method for Selection of Bayesian Chain Sampling Plan based on range of quality instead of point wise description of quality by invoking a novel approach called Quality Interval Sampling (QIS) plan. This method seems to be versatile and can be adopted in the elementary production process where the stipulated quality level is advisable to fix at later stage and provides a new concept for Selection of Bayesian Chain Sampling Plan (ChSP-1) involving quality levels.

The sampling plan provides both vendor and buyer decision rules for the product acceptance to meet the present product quality requirement. Due to rapid advancement of manufacturing technology, suppliers require their products to be of high quality with very low fraction defectives often measured in parts per million. Unfortunately, traditional methods in some particular situations fail to find out a minute defect in the product. In order to overcome such problems Quality Interval Sampling (QIS) plan is introduced. This paper designs the parameters for the plan indexed with quality regions involving QIS.

Case and Keats (1982) have examined the relationship between defectives in the sample and defectives in the remaining lot for each of the five prior distributions. They observe that the use of a binomial prior renders sampling useless and inappropriate. These results serve to make the designers and users of Bayesian Sampling Plans more aware of the consequence associated with selection of particular prior distribution. Calvin (1984) has presented in a clear and concise treatment by means of 'How and When to perform Bayesian acceptance Sampling'. These procedures are suited to the sampling of lots from process or assembly operations, which contain assignable causes. These causes may be unknown and awaiting isolation, known but irremovable due to the state of the art limitations, or known but uneconomical to remove. He has considered the Bayesian sampling, in which, primary concern is with the process average function non-conforming $\mathrm{p}$, with lot fraction non-conforming $\mathrm{p}$ and its limitations being discussed.

Hald (1960) has derived optimal solutions for the cost function $\mathrm{k}(\mathrm{n}, \mathrm{c})$ in the cases where the prior distribution is rectangular, polya and binomial. Tables are given for optimum $n, c$ and $k(n, c)$ for various values of the parameters, which is an important result on Bayesian Acceptance Sampling(BAS). Hald (1965) has given a rather complete tabulation and discussed the properties of a system of single Sampling attribute plans obtained by minimizing average costs, under the assumptions that the costs linear in the fraction defective $p$, and that the distributions of lot quality is a double binomial distribution. The optimum sampling plan (n.c) depends on six parameters namely $\mathrm{N}, \mathrm{p}_{\mathrm{r}}, \mathrm{p}_{\mathrm{s}}, \mathrm{p}_{1}, \mathrm{p}_{2}$ and $\mathrm{w}_{2}$ cost parameters and $\mathrm{p}_{1}, \mathrm{p}_{2}, \mathrm{w}_{2}$ are the parameters of prior distribution. It may be shown, however, that the weights combine with the p's is such a way that only five independent parameters are left out.

A set of tables presented by Oliver and Springer (1972) are based on assumptions of beta prior distribution with specific posterior risk to achieve minimum sample size, which avoids the problem of estimating cost parameters. It is generally true that Bayesian Plan requires a smaller sample size than a conventional sampling plan with the same producer and consumer risks. Schafer (1967) discusses single sampling by attributes using three prior distributions of lot quality. The desirability for developing a set of sampling plans indexed with $\mathrm{p} *$ has been explained by Mandelson (1962). Mayer (1967) has suggested that MAPD can be considered as a quality level, along with certain other conditions to specify OC curve.

Suresh and Latha (2002) has studied average probability of acceptance function for chain sampling plan with Gamma Prior distribution. Formula of inflection point and tangent at the inflection point are also derived. A selection procedure for Bayesian Chain sampling attributes plan (with Gamma prior distribution) based on AQL and LQL, point of control and relative slope at that point, MAAPD and $\mathrm{K}$, measure of sharpness are also explained, and lot acceptance procedures are developed for Bayesian Chain sampling attributes plans when the acceptance number is fixed and when the sample size is fixed. Suresh and Deepa (2002) has studied the formulation of a Bayesian Sampling Plan using acceptance probability with Gamma prior distribution for product quality using producer and consumer quality levels with selection procedure for Bayesian Special type of Double Sampling Plan with MAAOQ and also the sum of weighted risks and designing sampling plans of the one plan suspension system and Quick switching system. 
Suresh and Pradeepa veerakumari (2007) have given a procedure and tables for the selection of for Bayesian Chain Sampling Plan. Suresh and Kaviyarasu (2008) have explained the desirability for developing Quick Switching System with Conditional RGS plan indexed through quality levels. Suresh and Divya (2009) have given the new procedure for Single Sampling Plan through Decision Regions. Suresh and Sangeetha (2010) have studied the selection of Repetitive Deferred Sampling plan with Quality Regions. This paper designs the parameters of the plan indexed with QDR, PQR, LQR and IQR with numerical illustrations are also provided.

\section{Bayesian Chain Sampling Plan}

This paper related to Bayesian Chain Sampling Plan for average probability function of incoming quality levels.

\subsection{Chain Sampling Plan (ChSP-1)}

Sampling inspection in which the criteria for acceptance and non acceptance of the lot depend in part on the results of the inspection of immediately preceding lots is adopted in Chain Sampling Plan. Chain Sampling Plan (ChSP-1) proposed by Dodge (1955) making use of cumulative results of several samples help to overcome the short comings of the Single Sampling Plan.

\subsection{Conditions for application of ChSP -1:}

The cost of destructiveness of testing is such that a relatively small sample sizes i necessary, although other factors make a large sample desirable.

1. The product to be inspected comprises a series of successive lots produced by a continuing process.

2. Normally lots are expected $t$ be of essentially the same quality.

3. The consumer has faith in the integrity of the producer.

\subsection{Operating Procedure}

The plan is implemented in the following way:

1. For each lot, select a sample of $n$ units and test each unit for conformance to the specified requirements.

2. Accept the lot if $d$ (the observed number of defectives) is zero in the sample of $n$ units, and reject if $d>$ 1.

3. Accept the lot if $d$ is equal to 1 and if no defectives are found in the immediately preceding i samples of size $n$.

Dodge (1955) has given the operating characteristic function of ChSP-1 as

$$
\mathrm{P}_{\mathrm{a}}(\mathrm{p})=\mathrm{P}_{0}+\mathrm{P}_{1}\left(\mathrm{P}_{0}\right)^{\mathrm{i}}
$$

Where $P_{j}=$ probability of finding $j$ nonconforming units in a sample of $n$ units for $j=0.1$.

The Chain sampling Plan is characterized by the parameters $n$ and $i$. When $i=\infty$, the OC function of a ChSP-1 plan reduces to the OC function of the Single Sampling Plan with acceptance number zero and when $\mathrm{i}=0$, the OC function of ChSP-1 plan reduces to the OC function of the Single Sampling Plan with acceptance number 1.

\subsection{Bayesian Chain Sampling Plan (BChSP-1)}

According to Dodge (1955) the operating characteristic function of ChSP-1 is

$\mathrm{Pa}(\mathrm{p})=\mathrm{P}_{0}+\mathrm{P}_{1}\left(\mathrm{P}_{0}\right)^{\mathrm{i}}$,

The Chain Sampling Plan (ChSP-1) is characterized by two parameters $n$ and $i$, where $n$ is the sample size and $i$ is the number of preceding samples with zero defective, using the OC curve, Dodge (1955) has studied the properties of the Chain Sampling Plan. Clark (1960) has presented additional OC curves, which cover most of the situations. Soundararajan (1978 a, b) has described procedures and tables for construction and selection of Chain sampling plans (ChSP-1) indexed by specified parameters.

The probability of acceptance of ChSP-1 based on Poisson Model is provided as

$$
P(n, i / p)=e^{-n p}+e^{-n p(1+i)} n p
$$

Using the Past history of inspection, it is observed that $\mathrm{p}$ follows Gamma distribution

with density function,

$$
w(p)=e^{-p t} p^{s-i} t^{s} / \mathrm{g}(s), s, t>0, p>0
$$

The average probability of acceptance is given as

$\bar{P}=\int_{0}^{\infty} P(n, i / p) w(p) d p$ 


$$
\bar{P}=\frac{s^{s}}{(s+n \mu)^{s}}+\frac{n \mu s^{s+1}}{(s+n \mu+i n \mu)^{s+1}}
$$

Where $\mu=s / t$, is the mean value of the product quality $\mathrm{p}$.

\section{Selection of Bayesian Chain Sampling Plan}

\section{Designing of Quality Interval Bayesian chain Sampling Plan (QIBChsp-1)}

\subsection{QUALITY DECISION REGION $(Q D R)$}

It is an interval of quality $\left(\mu_{1}<\mu<\mu_{*}\right)$ in which product is accepted at engineer's quality average. The quality is reliably maintained up to $\mu_{*}$ (MAPD) and sudden decline in quality is expected. This region is also called Reliable Quality Region (RQR).

Quality decision Range is denoted as $d_{1}=\left(\mu_{*}-\mu_{1}\right)$ is derived from the average probability of acceptance

$$
\bar{P}\left(\mu_{1}<\mu<\mu_{*}\right)=s^{s} /(s+n \mu)+n s^{s+1} \mu /(s+n \mu+n i \mu)^{s+1} \text { for } \quad \mu_{1}<\mu<\mu_{*}
$$

where $\mu=\frac{s}{t}$, is the mean value for the product quality $\mathrm{p}$.

\subsection{PROBABILISTIC QUALITY REGION (PQR)}

It is an interval of quality $\left(\mu_{1}<\mu<\mu_{2}\right)$ in which product is accepted with a minimum probability 0.10 and maximum probability 0.95 .

Probabilistic Quality Range denoted as $d_{2}=\left(\mu_{2}-\mu_{1}\right)$ is derived from the average probability of acceptance $\bar{P}\left(\mu_{1}<\mu<\mu_{2}\right)=s^{s} /(s+n \mu)+n s^{s+1} \mu /(s+n \mu+n i \mu)^{s+1}$ for $\quad \mu_{1}<\mu<\mu_{2}$

where $\mu=\frac{s}{t}$, is the mean value of the product quality $\mathrm{p}$.

\subsection{LIMITING QUALITY REGION (LQR)}

It is an interval of quality $\left(\mu_{*}<\mu<\mu_{2}\right)$ in which product is accepted with a minimum probability 0.10 and maximum probability 0.95 .

Limiting Quality Range denoted as $d_{3}=\left(\mu_{2}-\mu_{*}\right)$ is derived from the average probability of acceptance

$$
\bar{P}\left(\mu_{*}<\mu<\mu_{2}\right)=s^{s} /(s+n \mu)+n s^{s+1} \mu /(s+n \mu+n i \mu)^{s+1} \text { for } \quad \mu_{*}<\mu<\mu_{2}
$$

where $\mu=\frac{s}{t}$, is the mean value of the product quality $\mathrm{p}$.

\subsection{Indifference Quality Region (IQR)}

It is an interval of quality $\left(\mu_{1}<\mu<\mu_{0}\right)$ in which product is accepted with a minimum probability 0.50 and maximum probability 0.95 .

Indifference Quality Range denoted as $d_{0}=\left(\mu_{0}-\mu_{1}\right)$ is derived from the average probability of acceptance $\bar{P}\left(\mu_{1}<\mu<\mu_{0}\right)=s^{s} /(s+n \mu)+n s^{s+1} \mu /(s+n \mu+n i \mu)^{s+1}$ for $\quad \mu_{1}<\mu<\mu_{0}$

where $\mu=\frac{s}{t}$, is the mean value of the product quality $\mathrm{p}$.

\subsection{Selection of the Sampling plan}

Table (1) shows the value of $\mathrm{s}, \mathrm{m}$ and corresponding ranges $\mathrm{d}_{1}=\mathrm{nQDR}, \mathrm{d}_{2}=\mathrm{nPQR}, \mathrm{d}_{3}=\mathrm{nLQR}$ and $\mathrm{d}_{0}=\mathrm{nIQR}$. Define a ratio $\quad T=\frac{\mu_{*}-\mu_{1}}{\mu_{2}-\mu_{1}}=\frac{\left(n \mu_{*}-n \mu_{1}\right)}{\left(n \mu_{2}-n \mu_{1}\right)} \quad, \quad T_{1}=\frac{\mu_{*}-\mu_{1}}{\mu_{2}-\mu_{*}}=\frac{\left(n \mu_{*}-n \mu_{1}\right)}{\left(n \mu_{2}-n \mu_{*}\right)}$ $T_{2}=\frac{\mu_{*}-\mu_{1}}{\mu_{0}-\mu_{1}}=\frac{\left(n \mu_{*}-n \mu_{1}\right)}{\left(n \mu_{0}-n \mu_{1}\right)}$ which is used to characterize the sampling plan. For any given values of 
QDR $\left(\mathrm{d}_{1}\right)$, PQR $\left(\mathrm{d}_{2}\right), \mathrm{LQR}\left(\mathrm{d}_{3}\right)$ and IQR $\left(\mathrm{d}_{0}\right)$. One can find the ratio $T=\frac{d_{1}}{d_{2}}, T_{1}=\frac{d_{1}}{d_{3}}$ and $T_{2}=\frac{d_{1}}{d_{0}}$. Find the value in the Table(1) under the column $T, T_{1}$ and $T_{2}$, which is equal to or just less than the specified ratio, corresponding $\mathrm{s}$ and $\mathrm{i}$, values are noted. From this ratio, one can determine the parameters for Bayesian Chain Sampling Plan.

\subsubsection{Numerical Example}

Given $\mu_{1}=0.01, \mathrm{~s}=5$ and $\mathrm{i}=2$ compute the values of QDR, PQR, LQR and IQR. Then compute $\mathrm{T}, \mathrm{T}_{1}$ and $\mathrm{T}_{2}$ selects the respective values from Table1. The nearest values are $\mathrm{QDR}=0.1953$. $\mathrm{PQR}=2.8707$, $\mathrm{LQR}=2.6754, \mathrm{IQR}=0.7564$, and the ratio $\mathrm{T}=0.0680, \mathrm{~T}_{1}=0.0730, \mathrm{~T}_{2}=0.2582$ with respect to $\mathrm{s}$ and $\mathrm{m}$. Corresponding $\mathrm{s}$ and $\mathrm{m}$, one can obtain the values of $n \mu_{1}$ from Table2, which is $n \mu_{1}=0.1538$. From this one can obtain $\mathrm{n}=\mathrm{n} \mu_{1} / \mu_{1}=0.1538 / 0.01 \approx 16$ using Table1,

Thus the selected parameters for Bayesian Chain Sampling Plan are $n=16, s=5$ and $i=2(\operatorname{BChSP}-1(16,5,2))$ through quality interval.

\subsection{For specified $Q D R$ and $P Q R$}

Table 1 is used to construct the plans when the QDR and PQR are specified. For any given values of the QDR $\left(d_{1}\right)$ and PQR $\left(d_{2}\right)$, one can find the ratio $T=d_{1} / d_{2}$ which is a monotonic increasing function. Find the value in Table 1 under the column $\mathrm{T}$ which is equal to or just less than the specified ratio. Then the corresponding values of $s$ and $i$ are noted. From this, one can determine the parameters n, s and i for the Bayesian Chain Sampling Plan.

\subsubsection{Example}

For a company $1 \%$ defects are seen in QDR and 9\% defects are seen in PQR.

Then $\mathrm{d}_{1}=0.01$ and $\mathrm{d}_{2}=0.09, \mathrm{~T}=\mathrm{d}_{1} / \mathrm{d}_{2}=0.112$

Find the ratio taking value 0.112 . Select value of $\mathrm{T}$ equal to or just less than this ratio using Table 1 . The value of $\mathrm{T}$ is 0.1129 which is associated with $\mathrm{s}=7$ and $\mathrm{i}=1$. Also $\mathrm{nd}_{1}=0.3249, \mathrm{nd}_{2}=2.8787$ corresponding to $\mathrm{s}=7$ and $\mathrm{i}=1$. Thus $\mathrm{n}$ is calculated. The parameters for the Bayesian Chain Sampling Plan is $(33,7,1)$.

\subsection{For specified $Q D R$ and $L Q R$}

Table 1 is used to construct the plans when the QDR and LQR are specified. For any given values of the QDR $\left(d_{1}\right)$ and LQR $\left(d_{3}\right)$, one can find the ratio $T_{1}=d_{1} / d_{3}$ which is a monotonic increasing function. Find the value in Table 1 under the column $T_{1}$ which is equal to or just less than the specified ratio. Then the corresponding values of $\mathrm{s}$ and $\mathrm{i}$ are noted. From this, one can determine the parameters $\mathrm{n}, \mathrm{s}$ and $\mathrm{i}$, for the Bayesian Chain Sampling Plan.

\subsubsection{Example}

For a company $1 \%$ defects are seen in QDR and $7 \%$ defects are seen in LQR.

Then $\mathrm{d}_{1}=0.01$ and $\mathrm{d}_{3}=0.07, \mathrm{~T}_{1}=\mathrm{d}_{1} / \mathrm{d}_{3}=0.143$.

Find the ratio taking value 0.143 . Select value of $T_{1}$ equal to or just less than this ratio using Table 1. The value of $\mathrm{T}_{1}$ is 0.1500 which is associated with $\mathrm{s}=7$ and $\mathrm{i}=0$. Also $\mathrm{nd}_{1}=0.5870, \mathrm{nd}_{3}=3.9144$ corresponding to $\mathrm{s}=7$ and $\mathrm{i}=0$. Thus $\mathrm{n}$ is calculated. The parameters for the Bayesian Chain Sampling Plan is $(59,7,0)$.

\subsection{For specified $Q D R$ and $I Q R$}

Table 1 is used to construct the plans when the QDR and IQR are specified. For any given values of the QDR $\left(\mathrm{d}_{1}\right)$ and IQR $\left(\mathrm{d}_{0}\right)$, one can find the ratio $\mathrm{T}_{2}=\mathrm{d}_{1} / \mathrm{d}_{0}$ which is a monotonic increasing function. Find the value in Table 1 under the column $T_{2}$ which is equal to or just less than the specified ratio. Then the corresponding values of $\mathrm{s}$ and $\mathrm{i}$ are noted. From this, one can determine the parameters $\mathrm{n}, \mathrm{s}$ and $\mathrm{i}$, for the Bayesian Chain Sampling Plan.

\subsubsection{Example}

For a company $2 \%$ defects are seen in QDR and $8 \%$ defects are seen in IQR.

Then $\mathrm{d}_{1}=0.02$ and $\mathrm{d}_{0}=0.08, \mathrm{~T}_{2}=\mathrm{d}_{1} / \mathrm{d}_{0}=0.25$ 
Find the ratio taking value 0.25 . Select value of $\mathrm{T}_{2}$ equal to or just less than this ratio using Table 1. The value of $\mathrm{T}_{2}$ is 0.2481 which is associated with $\mathrm{s}=3$ and $\mathrm{i}=1$. Also $\mathrm{nd}_{1}=0.2354, \mathrm{nd}_{0}=0.9488$ corresponding to $\mathrm{s}=3$, and $\mathrm{i}=1$. Thus $\mathrm{n}$ is calculated. The parameters for the Bayesian Chain Sampling Plan is $(12,3,1)$.

\subsection{For specified $A Q L$ and $L Q L$}

Table 2 is used to construct the plans when the $\mathrm{AQL}=\mu_{1}, \mathrm{LQL}=\mu_{2}$ and $\mathrm{s}$ are specified. For any given values of the AQL $\left(\mu_{1}\right)$ and LQL $\left(\mu_{2}\right)$, the $\mathrm{OR}=\mu_{2} / \mu_{1}$ is calculated. The values of $m$ and $n \mu_{1}$ corresponding to the specified OR and $s$ are found out. The sample size $n$ is obtained by dividing $n \mu_{1}$ by $\mu$. From this, one can determine the parameters n, s and i for the Bayesian Chain Sampling Plan

\subsubsection{Example}

It is given that $\mathrm{s}=1$ at $\alpha=0.05, \mu_{1}=0.1 \%$ and $\beta=0.10, \mu_{2}=9.5 \%$

Then the operating ratio OR $=\mu_{2} / \mu_{1}=95$. The value of OR in table 2 nearer to 95 is 95.3528 for which $n \mu_{1}=$ 0.0975 and hence $\mathrm{n}$ is calculated as equal to $97.5 \approx 98$. The parameters for the Bayesian Chain Sampling Plan is $(98,1,5)$.

\subsection{Conversion of parameters}

The given set of parameters can be converted into another set, which will provide related information on the derived sampling plan. These parameters can be found using Table 2.

\subsubsection{Example}

For given $\mu_{1}=0.01, \mathrm{~s}=5$ and $\mathrm{i}=2$ compute the values of QDR, PQR, LQR and IQR. Corresponding $\mathrm{s}=5$ and $\mathrm{i}=2$ values of $\mathrm{QDR}=0.1953 . \mathrm{PQR}=2.8707, \mathrm{LQR}=2.6754, \mathrm{IQR}=0.7564$, and the ratio $\mathrm{T}=0.0680, \mathrm{~T}_{1}=0.0730, \mathrm{~T}_{2}=$ 0.2582 with respect to $\mathrm{s}$ and $\mathrm{i}$. And the parameters are $\mathrm{n}=11, \mathrm{~s}=5$ and $\mathrm{i}=2(\operatorname{BChSP}-1(16,5,2))$ using Table2.

The other set of parameters are as follows:

1) $n \mu_{1}=0.1538, n \mu_{2}=3.0245$ and $n \mu_{*}=0.3491$,

2) $n \mu_{0}=0.9102, n \mu_{m}=0.9190$,

3) $\mathrm{h}_{0}=0.8166, \mathrm{~h}_{*}=0.2875$

\section{Construction of Tables}

The probability of acceptance of ChSP-1 based on Poisson Model is provided as

$$
P(n, i / p)=e^{-n p}+e^{-n p(1+i)} n p
$$

The average probability of acceptance is given as

$$
\bar{P}=\int_{0}^{\infty} P(n, i / p) w(p) d p
$$

On simplification, the APA function for Chsp-1

$$
\bar{P}=\frac{s^{s}}{(s+n \mu)^{s}}+\frac{n \mu s^{s+1}}{(s+n \mu+i n \mu)^{s+1}}
$$

Where $\mu=s / t$, is the mean value of the product quality $\mathrm{p}$.

Tables 1 shows the value of $s$ and $i$ corresponding ranges $d_{1}=n Q D R, d_{2}=n P Q R, d_{3}=n L Q R$ and $d_{0}=n I Q R$ from equation (3.1), (3.2), (3.3) and (3.4), and also represents Operating Characteristic ratio for specified values of $\mathrm{s}$ and i. Table 2 represents the conversion table, which is used to determine other quality characteristics.

\section{Conclusion}

Bayesian Acceptance sampling is the technique, which deals with the procedures in which decision to accept or reject lots or process based on their examination of past history or knowledge of samples. This paper deals with sampling model based on prior distribution and costs, which encompasses most of the existing Bayesian models 
based on costs. The work is presented in this paper mainly related to construction and selection Bayesian Chain Sampling Plan(ChSP-1) for Quality Regions.

Quality Interval Sampling (QIS) plan possesses wider potential applicability in industry ensuring higher standard of quality attainment for product or process. Thus Quality Interval Sampling (QIS) plan is a good measure for defining quality and designing any acceptance sampling plan which are readymade use to industrial shop-floor situations.

The Quality Decision Region (QDR) idea is proposed in order to provide higher probability of acceptance compared with (AQL, LQL) indexed plan/scheme/system. Quality Decision Region (QDR) depends on the quality measure MAPD, which is a key measure assessing to what degree the inflection point empowers the OC curve to discriminate between good and bad lots. The present development would be a valuable addition to the literature and a useful device for quality control practitioners.

This paper mainly relates to the construction and selection of performance measures for Quality Interval Sampling (QIS) inspection plan indexed through Quality Regions. Conversions of parameters are also provided for comparison. Tables are provided which are tailor-made, handy and ready-made uses to the industrial shop floor situations.

\section{References}

Calvin, T.W. (1984). How and When to perform Bayesian Acceptance Sampling, Vol.7, American Society for Quality Control, Milwaukee, Wisconsin.

Case, K.E and Keats, J.B. (1982). On the selection of a Prior Distribution in Bayesian Acceptance Sampling, Journal of Quality Technology, Vol.14(1), 10-18.

Clark, C. R. (1960). OC Curve for ChSP-1 Chain Sampling Plans, Industrial Quality Control, Vol.17, No. 4, pp.10-12.

Dodge, H.F. (1955). Chain Sampling Inspection Plan, Industrial Quality Control, Vol.11, No.4, pp.10-13.

Hald, A. (1960). The Compound Hypergeometric Distribution and a system of Single Sampling Inspection Plans based on Prior Distributions and Costs, Technometrics, Vol.2, pp.275-340.

Hald, A. (1965). Bayesian Single Sampling Plans for Discrete Prior Distributions, Mat. Fys. Skr. Dan. Vid. Selsk., Vol.3 (2), Munksgaard, Copenhegan.

Mandelson, J. (1962). The Statistician, the Engineer and Sampling plans, Industrial Quality Control, vol.19, pp.12-15.

Mayer, P.L. (1967). A note on the sum of Poisson probabilities and its Applications, Annals of the Institute of statistical Mathematics, Vol.19, pp. 537-542.

Oliver, L.R and Springer, M.D. (1972). A General Set of Bayesian Attribute Acceptance Plans, American Institute of Industrial Engineers, Norcross, G.A.

Scafer, R.E. (1967). Bayes Single Sampling Plans by Attributes Based on the Posterior Risks, Navel Research Logistics Quaterly, Vol.14, No.1, pp.81-88.

Soundararajan, V. (1978a,b). Procedure and Tables for Construction and Selection of Chain Sampling Plan(ChSP-1), Part I and II, Journal of Quality Technology, Vol.10, No.2, pp.56-60 and No.3, pp.99-103.

Suresh K. K. and. Deepa O. S. (2002). Risk based Bayesian Chain Sampling Plan, Far East Journal of Theoretical Statistics, Vol. 6, No. 2, pp. $121-128$.

Suresh, K.K and Divya, P.R. (2009). Selection of Single Sampling Plan through Decision Region, International Journal of Applied Mathematics \& Statistics, Vol.14, No.S09, pp.66-78.

Suresh, K.K and Kaviyarasu, V. (2008). Certain Results and Tables Relating QSS-1 with Conditional RGS Plan, IAPQR Transactions, Vol.33, No.1, pp 61-70.

Suresh, K.K and Latha, M. (2002). Construction and Evaluation of Performance Measures for Bayesian Chain Sampling Plan (BChSP-1), Far East Journal of Theoretical Statistics, Vol. 6, No. 2, pp.129-139.

Suresh, K.K and Pradeepa Veerakumari. K, (2007). Construction and Evaluation of Performance Measures for Bayesian Chain Sampling Plan (BChSP-1), Acta Ciencia Indica, No. 4, Vol. 33, pp. 16-35.

Suresh, K.K and Sangeetha, V. (2010). Selection of Repetitive Deferred Sampling Plan through Quality Region, International Journal of Statistics and Systems, Vol. 5, No.3, pp.379-389. 
Table 1. Certain Values of QDR, PQR, LQR and IQR \& Operating Characteristic ratio for Specified values of s and $\mathrm{i}$.

\begin{tabular}{|c|c|c|c|c|c|c|c|c|}
\hline $\mathrm{s}$ & $\mathrm{i}$ & $\mathrm{nd}_{1}$ & $\mathrm{nd}_{2}$ & $\mathrm{nd}_{3}$ & $\mathrm{nd}_{0}$ & $\mathrm{~T}=\mathrm{d}_{1} / \mathrm{d}_{2}$ & $\mathrm{~T}_{1}=\mathrm{d}_{1} / \mathrm{d}_{3}$ & $\mathrm{~T}_{2}=\mathrm{d}_{1} / \mathrm{d}_{0}$ \\
\hline \multirow[t]{10}{*}{1} & 0 & 0.2120 & 18.1988 & 17.9868 & 2.1262 & 0.0116 & 0.0118 & 0.0997 \\
\hline & 1 & 0.1161 & 11.3271 & 11.2110 & 1.2989 & 0.0102 & 0.0104 & 0.0894 \\
\hline & 2 & 0.0804 & 10.0102 & 9.9298 & 1.1151 & 0.0080 & 0.0081 & 0.0721 \\
\hline & 3 & 0.0597 & 9.5391 & 9.4794 & 1.0411 & 0.0063 & 0.0063 & 0.0573 \\
\hline & 4 & 0.0458 & 9.3190 & 9.2732 & 1.0038 & 0.0049 & 0.0049 & 0.0456 \\
\hline & 5 & 0.0357 & 9.1994 & 9.1637 & 0.9834 & 0.0039 & 0.0039 & 0.0363 \\
\hline & 6 & 0.0280 & 9.1277 & 9.0997 & 0.9697 & 0.0031 & 0.0031 & 0.0289 \\
\hline & 7 & 0.0218 & 9.0814 & 9.0596 & 0.9593 & 0.0024 & 0.0024 & 0.0227 \\
\hline & 8 & 0.0168 & 9.0500 & 9.0332 & 0.9557 & 0.0019 & 0.0019 & 0.0176 \\
\hline & 9 & 0.0125 & 9.0278 & 9.0153 & 0.9518 & 0.0014 & 0.0014 & 0.0131 \\
\hline \multirow[t]{10}{*}{3} & 0 & 0.4255 & 6.0370 & 5.6115 & 1.5593 & 0.0705 & 0.0758 & 0.2729 \\
\hline & 1 & 0.2354 & 3.8300 & 3.5946 & 0.9488 & 0.0615 & 0.0655 & 0.2481 \\
\hline & 2 & 0.1671 & 3.4924 & 3.3253 & 0.8092 & 0.0478 & 0.0503 & 0.2065 \\
\hline & 3 & 0.1285 & 3.4077 & 3.2792 & 0.7555 & 0.0377 & 0.0392 & 0.1701 \\
\hline & 4 & 0.1031 & 3.3828 & 3.2797 & 0.7297 & 0.0305 & 0.0314 & 0.1413 \\
\hline & 5 & 0.0848 & 3.3758 & 3.2910 & 0.7167 & 0.0251 & 0.0258 & 0.1183 \\
\hline & 6 & 0.0710 & 3.3748 & 3.3038 & 0.7101 & 0.0210 & 0.0215 & 0.1000 \\
\hline & 7 & 0.0601 & 3.3761 & 3.3160 & 0.7069 & 0.0178 & 0.0181 & 0.0850 \\
\hline & 8 & 0.0512 & 3.3780 & 3.3268 & 0.7054 & 0.0152 & 0.0154 & 0.0726 \\
\hline & 9 & 0.0430 & 3.3802 & 3.3372 & 0.7051 & 0.0127 & 0.0129 & 0.0610 \\
\hline \multirow[t]{10}{*}{5} & 0 & 0.4980 & 4.8754 & 4.3774 & 1.4622 & 0.1021 & 0.1138 & 0.3406 \\
\hline & 1 & 0.2753 & 3.1126 & 2.8373 & 0.8882 & 0.0884 & 0.0970 & 0.3100 \\
\hline & 2 & 0.1953 & 2.8707 & 2.6754 & 0.7564 & 0.0680 & 0.0730 & 0.2582 \\
\hline & 3 & 0.1505 & 2.8249 & 2.6744 & 0.7045 & 0.0533 & 0.0563 & 0.2136 \\
\hline & 4 & 0.1209 & 2.8145 & 2.6936 & 0.6807 & 0.0430 & 0.0449 & 0.1776 \\
\hline & 5 & 0.0998 & 2.8211 & 2.7213 & 0.6694 & 0.0354 & 0.0367 & 0.1491 \\
\hline & 6 & 0.0838 & 2.8254 & 2.7416 & 0.6643 & 0.0297 & 0.0306 & 0.1261 \\
\hline & 7 & 0.0713 & 2.8298 & 2.7585 & 0.6624 & 0.0252 & 0.0258 & 0.1076 \\
\hline & 8 & 0.0610 & 2.8337 & 2.7727 & 0.6622 & 0.0215 & 0.0220 & 0.0921 \\
\hline & 9 & 0.0527 & 2.8372 & 2.7845 & 0.6629 & 0.0186 & 0.0189 & 0.0795 \\
\hline \multirow[t]{10}{*}{7} & 0 & 0.5870 & 4.5014 & 3.9144 & 1.4745 & 0.1304 & 0.1500 & 0.3981 \\
\hline & 1 & 0.3249 & 2.8787 & 2.5538 & 0.8922 & 0.1129 & 0.1272 & 0.3642 \\
\hline & 2 & 0.2315 & 2.6660 & 2.4345 & 0.7555 & 0.0868 & 0.0951 & 0.3064 \\
\hline & 3 & 0.1789 & 2.6504 & 2.4715 & 0.7004 & 0.0675 & 0.0724 & 0.2554 \\
\hline & 4 & 0.1443 & 2.6279 & 2.4836 & 0.6746 & 0.0549 & 0.0581 & 0.2139 \\
\hline & 5 & 0.1196 & 2.6314 & 2.5118 & 0.6621 & 0.0455 & 0.0476 & 0.1806 \\
\hline & 6 & 0.1009 & 2.6358 & 2.5349 & 0.6561 & 0.0383 & 0.0398 & 0.1538 \\
\hline & 7 & 0.0862 & 2.6396 & 2.5534 & 0.6535 & 0.0327 & 0.0338 & 0.1319 \\
\hline & 8 & 0.0743 & 2.6430 & 2.5687 & 0.6528 & 0.0281 & 0.0289 & 0.1138 \\
\hline & 9 & 0.0644 & 2.6458 & 2.5814 & 0.6531 & 0.0243 & 0.0249 & 0.0986 \\
\hline
\end{tabular}


Table 2. Certain Parametric Values of Bayesian Chain Sampling Plan (ChSP-1)

\begin{tabular}{|c|c|c|c|c|c|c|c|c|c|}
\hline $\mathrm{s}$ & $\mathrm{i}$ & $\mathrm{n} \mu_{1}$ & $\mathrm{n} \mu_{0}$ & $\mathrm{n} \mu_{2}$ & $n \mu *$ & $\mathrm{n} \mu_{\mathrm{m}}$ & $\mu_{2} / \mu_{1}$ & $\mathrm{~h}_{0}$ & $\mathrm{~h}_{*}$ \\
\hline \multirow[t]{9}{*}{1} & 0 & 0.2880 & 2.4142 & 18.4868 & 0.5000 & 1.5486 & 64.1903 & 0.5858 & 0.1667 \\
\hline & 1 & 0.1686 & 1.4675 & 11.4957 & 0.2847 & 1.0000 & 68.1833 & 0.5752 & 0.1575 \\
\hline & 2 & 0.1340 & 1.2491 & 10.1442 & 0.2144 & 0.9461 & 75.7030 & 0.5580 & 0.1419 \\
\hline & 3 & 0.1163 & 1.1574 & 9.6554 & 0.1760 & 0.9521 & 83.0215 & 0.5444 & 0.1281 \\
\hline & 4 & 0.1052 & 1.1090 & 9.4242 & 0.1510 & 0.9641 & 89.5837 & 0.5346 & 0.1167 \\
\hline & 5 & 0.0975 & 1.0809 & 9.2969 & 0.1332 & 0.9736 & 95.3528 & 0.5277 & 0.1073 \\
\hline & 6 & 0.0917 & 1.0614 & 9.2194 & 0.1197 & 0.9806 & 100.5387 & 0.5223 & 0.0995 \\
\hline & 7 & 0.0873 & 1.0466 & 9.1687 & 0.1091 & 0.9854 & 105.0252 & 0.5179 & 0.0928 \\
\hline & 8 & 0.0837 & 1.0394 & 9.1337 & 0.1005 & 0.9888 & 110.4438 & 0.5154 & 0.0871 \\
\hline & 9 & 0.0808 & 1.0326 & 9.1086 & 0.0933 & 0.9913 & 112.7302 & 0.5131 & 0.0821 \\
\hline \multirow[t]{10}{*}{3} & 0 & 0.3245 & 1.8838 & 6.3615 & 0.7500 & 1.5790 & 19.6040 & 0.8277 & 0.3000 \\
\hline & 1 & 0.1892 & 1.1380 & 4.0192 & 0.4246 & 1.0000 & 21.2431 & 0.8014 & 0.2794 \\
\hline & 2 & 0.1493 & 0.9585 & 3.6417 & 0.3164 & 0.9274 & 24.3918 & 0.7565 & 0.2457 \\
\hline & 3 & 0.1286 & 0.8841 & 3.5363 & 0.2571 & 0.9345 & 27.4987 & 0.7206 & 0.2170 \\
\hline & 4 & 0.1155 & 0.8452 & 3.4983 & 0.2186 & 0.9536 & 30.2883 & 0.6936 & 0.1940 \\
\hline & 5 & 0.1063 & 0.8230 & 3.4821 & 0.1911 & 0.9689 & 32.7573 & 0.6741 & 0.1753 \\
\hline & 6 & 0.0994 & 0.8095 & 3.4742 & 0.1704 & 0.9793 & 34.9517 & 0.6602 & 0.1601 \\
\hline & 7 & 0.0940 & 0.8009 & 3.4701 & 0.1541 & 0.9860 & 36.9160 & 0.6502 & 0.1474 \\
\hline & 8 & 0.0897 & 0.7951 & 3.4677 & 0.1409 & 0.9903 & 38.6589 & 0.6429 & 0.1366 \\
\hline & 9 & 0.0861 & 0.7912 & 3.4663 & 0.1291 & 0.9932 & 40.2590 & 0.6376 & 0.1265 \\
\hline \multirow[t]{10}{*}{5} & 0 & 0.3353 & 1.7975 & 5.2107 & 0.8333 & 1.5916 & 15.5404 & 0.9034 & 0.3571 \\
\hline & 1 & 0.1953 & 1.0835 & 3.3079 & 0.4706 & 1.0000 & 16.9375 & 0.8714 & 0.3305 \\
\hline & 2 & 0.1538 & 0.9102 & 3.0245 & 0.3491 & 0.9190 & 19.6651 & 0.8166 & 0.2875 \\
\hline & 3 & 0.1322 & 0.8367 & 2.9571 & 0.2827 & 0.9262 & 22.3684 & 0.7700 & 0.2517 \\
\hline & 4 & 0.1185 & 0.7992 & 2.9330 & 0.2394 & 0.9493 & 24.7848 & 0.7351 & 0.2231 \\
\hline & 5 & 0.1089 & 0.7783 & 2.9300 & 0.2087 & 0.9680 & 26.9054 & 0.7099 & 0.2004 \\
\hline & 6 & 0.1017 & 0.7660 & 2.9271 & 0.1855 & 0.9800 & 28.7819 & 0.6921 & 0.1819 \\
\hline & 7 & 0.0960 & 0.7584 & 2.9258 & 0.1673 & 0.9874 & 30.4771 & 0.6795 & 0.1666 \\
\hline & 8 & 0.0915 & 0.7537 & 2.9252 & 0.1525 & 0.9919 & 31.9694 & 0.6708 & 0.1537 \\
\hline & 9 & 0.0877 & 0.7506 & 2.9249 & 0.1404 & 0.9947 & 33.3512 & 0.6646 & 0.1429 \\
\hline \multirow[t]{10}{*}{7} & 0 & 0.2880 & 1.7625 & 4.7894 & 0.8750 & 1.5979 & 14.0658 & 0.9409 & 0.3889 \\
\hline & 1 & 0.1686 & 1.0608 & 3.0473 & 0.4935 & 1.0000 & 15.3671 & 0.9058 & 0.3586 \\
\hline & 2 & 0.1340 & 0.8895 & 2.8000 & 0.3655 & 0.9142 & 17.9487 & 0.8455 & 0.3104 \\
\hline & 3 & 0.1163 & 0.8167 & 2.7667 & 0.2952 & 0.9214 & 20.5131 & 0.7935 & 0.2702 \\
\hline & 4 & 0.1052 & 0.7798 & 2.7331 & 0.2495 & 0.9471 & 22.7758 & 0.7540 & 0.2385 \\
\hline & 5 & 0.0975 & 0.7596 & 2.7289 & 0.2171 & 0.9679 & 24.7856 & 0.7257 & 0.2134 \\
\hline & 6 & 0.0917 & 0.7478 & 2.7275 & 0.1926 & 0.9809 & 26.5321 & 0.7056 & 0.1931 \\
\hline & 7 & 0.0873 & 0.7408 & 2.7269 & 0.1735 & 0.9886 & 28.1124 & 0.6918 & 0.1764 \\
\hline & 8 & 0.0837 & 0.7365 & 2.7267 & 0.1580 & 0.9931 & 29.5417 & 0.6823 & 0.1624 \\
\hline & 9 & 0.0808 & 0.7339 & 2.7266 & 0.1452 & 0.9957 & 30.8439 & 0.6758 & 0.1506 \\
\hline
\end{tabular}

